\title{
The distribution and asympotic behaviour of the negative Wiener-Hopf factor for Lévy processes with rational positive jumps
}

\author{
EKaterina T. KolKovskA* Ehyter M. Martín-GonZÁLEZ ${ }^{\dagger}$
}

\begin{abstract}
We study the distribution of the negative Wiener-Hopf factor for a class of two-sided jumps Lévy processes whose positive jumps have a rational Laplace transform. The positive Wiener-Hopf factor for this class of processes was studied by Lewis and Mordecki [2008]. Here we obtain a formula for the Laplace transform of the negative Wiener-Hopf factor, as well as an explicit expression for its probability density, which is in terms of sums of convolutions of known functions. Under additional regularity conditions on the Lévy measure of the studied processes, we also provide asymptotic results as $u \rightarrow-\infty$ for the distribution function $F(u)$ of the negative Wiener-Hopf factor. We illustrate our results in some particular examples.

Keywords and phrases: Two-sided jumps Lévy process, Wiener-Hopf factorization, Negative WienerHopf factor, Lévy risk processes.
\end{abstract}

\section{Introduction}

The Wiener-Hopf factorization for Lévy processes has become a very important tool due to its applications in several branches of applied probability, such as insurance mathematics, theory of branching processes, mathematical finance and optimal control. For instance, when the market is modelled by a Lévy process, the positive Wiener-Hopf factor allows to solve the optimal stopping problem corresponding to the pricing of a perpetual call option, while the negative Wiener-Hopf factor is used to solve the optimal stopping problem corresponding to the pricing of a perpetual put option. This negative Wiener-Hopf factor also arises in insurance mathematics in connection with scale functions appearing in fluctuation identities. Such identities allow to obtain the joint distribution of the first passage time below a certain level and the position of the process at this time, which is the classical ruin problem.

For a one-dimensional Lévy process $\mathcal{X}=\{\mathcal{X}(t), t \geq 0\}$ we denote $S_{t}=\sup _{0 \leq s \leq t} \mathcal{X}(s)$ and $I_{t}=$ $\inf _{0 \leq s \leq t} \mathcal{X}(s)$. The explicit distribution of $S_{t}$ and $I_{t}$ in general is difficult to obtain but the following relation holds. Let $e_{q}$ be an independent exponential random variable with parameter $q>0$. The positive and negative Wiener-Hopf factors of $\mathcal{X}$ are defined respectively as the random variables $S_{e_{q}}$ and $I_{e_{q}}$ and they satisfy the identity

$$
\mathbb{E}\left[e^{i r S_{e_{q}}}\right] \mathbb{E}\left[e^{i r I_{e_{q}}}\right]=\frac{q}{q+\psi_{\mathcal{X}}(r)}, \quad r \in \mathbb{R},
$$

\footnotetext{
* Área de Probabilidad y Estadística, Centro de Investigación en Matemáticas, Guanajuato, Mexico.

${ }^{\dagger}$ Departamento de Matemáticas, Universidad de Guanajuato, Guanajuato, Mexico.
} 
where $\psi_{\mathcal{X}}(r)=-\log \mathbb{E}\left[e^{i r \mathcal{X}(1)}\right]$ is the characteristic exponent of $\mathcal{X}$. Only a few results are known for the explicit distribution of both Wiener-Hopf factors for processes with positive and negative jumps, see e.g. Feller [1971], Borovkov [1976], Asmussen et al. [2004] and Kuznetsov [2010a], Kuznetsov [2010b]. While the distribution of the positive Wiener-Hopf factor has been studied recently by several authors under some rather general conditions on the positive jumps (see, e.g. Kuznetsov [2010a], Kuznetsov and Peng [2012], Lewis and Mordecki [2008] and the references therein), the distribution of the negative factor in these cases has not be obtained explicitly.

In this paper we consider Lévy processes $\mathcal{X}$ with two-sided jumps such that the positive jumps have rational Laplace transform, and with general negative jumps. This class of Lévy processes has been studied recently in Lewis and Mordecki [2008], where the authors obtained the explicit distribution of the positive WienerHopf factor as well as asymptotic results for the tail of $S_{\infty}$. The particular case of Lévy processes with positive jumps which have phase-type distribution has been studied by Asmussen et al. [2004] where the authors obtained the distributions of both Wiener-Hopf factors. The class of distributions having rational Laplace transforms is rich enough since it it dense in the class of nonnegative distributions. By inverting the Laplace transform of the random variable $-I_{e_{q}}$ we provide an explicit expression for the probability density of the negative Wiener-Hopf factor $I_{e_{q}}$ in terms of given functions. Under additional regularity assumptions on the Lévy measure of $\mathcal{X}$ we obtain asymptotic results as $u \rightarrow-\infty$ for the distribution function $F(u)$ of the negative Wiener-Hopf factor $I_{e_{q}}$. Our formula for the density of the negative Wiener-Hopf factor generalizes the corresponding result in Asmussen et al. [2004].

The paper is organized as follows: in Section 2 we introduce basic concepts and notations and give some preliminary results. In Section 3 we obtain an expression for the Laplace transform of $-I_{e_{q}}$, which we invert in order to get an explicit formula for its probability density. In Section 4 we derive asymptotic results for the distribution of the negative Wiener-Hopf factor, while some relevant examples are given in Section 5 . In the final section we give the proof of the auxiliary Lemma 5.

\section{Preliminary results}

We consider the class of two-sided jumps Lévy processes $\mathcal{X}=\{\mathcal{X}(t), t \geq 0\}$, where

$$
\mathcal{X}(t)=c t+\gamma \mathcal{B}(t)+\mathcal{Z}(t)-\mathcal{S}(t), t \geq 0
$$

In the above expression, $c \geq 0$ is a drift term, $\mathcal{B}=\{\mathcal{B}(t), t \geq 0\}$ is a standard Brownian motion with variance parameter $2, \mathcal{S}=\{\mathcal{S}(t), t \geq 0\}$ is a pure jump Lévy process having only positive jumps and $\mathcal{Z}=\{\mathcal{Z}(t), t \geq 0\}$ is a compound Poisson process with Lévy measure $\lambda_{1} f_{1}(x) d x$, where $\lambda_{1}>0$ is constant. The function $f_{1}$ is assumed to be a probability density with Laplace transform of the form

$$
\widehat{f_{1}}(r)=\frac{Q(r)}{\prod_{i=1}^{N}\left(\alpha_{i}+r\right)^{n_{i}}}
$$


where $N, n_{i} \in \mathbb{N}$ with $n_{1}+n_{2}+\cdots+n_{N}=m, 0<\alpha_{1}<\alpha_{2}<\ldots \alpha_{N}$ are real numbers and $Q(r)$ is a polynomial of degree at most $m-1$. Let $\Psi_{\mathcal{S}}(r)=-\log \mathbb{E}\left[e^{-r \mathcal{S}(1)}\right]$ be the Laplace exponent of the process $\mathcal{S}$. It is known (see Sato [1999]) that $\Psi_{\mathcal{S}}(r)=\int_{0+}^{\infty}\left(1-e^{-r x}-r x h(x)\right) \nu_{\mathcal{S}}(d x)$, where $h$ is a truncation function and $\nu_{\mathcal{S}}$ is the Lévy measure of $\mathcal{S}$, which satisfies $\int_{0+}^{\infty}\left(x^{2} \wedge 1\right) \nu_{\mathcal{S}}(d x)<\infty$. We also set $\overline{\mathcal{V}}_{\mathcal{S}}(u)=\int_{u}^{\infty} \nu_{\mathcal{S}}(d x)$.

For $\mathcal{X}$ given in (2.1) we consider the function

$$
\Psi_{\mathcal{X}}(r)=c r+\gamma^{2} r^{2}+\lambda_{1}\left(\frac{Q(-r)}{\prod_{j=1}^{N}\left(\alpha_{j}-r\right)^{n_{j}}}-1\right)-\Psi_{\mathcal{S}}(r)
$$

Note that, for $0 \leq r<\alpha_{1}, \Psi_{\mathcal{X}}(r)=\log \mathbb{E}\left[e^{r \mathcal{X}(1)}\right]$.

When $\mathcal{S}$ is a subordinator we replace $\Psi_{\mathcal{S}}(r)$ in the above expression by $G_{\mathcal{S}}(r)=\int_{0+}^{\infty}\left(1-e^{-r x}\right) \nu_{\mathcal{S}}(d x)$, and assume that the drift term $c$ includes the constant $\int_{0+}^{\infty} x h(x) \nu_{\mathcal{S}}(d x)$.

In what follows we consider the sets $\mathbb{C}_{+}=\{z \in \mathbb{C}: \operatorname{Re}(z) \geq 0\}$ and $\mathbb{C}_{++}=\{z \in \mathbb{C}: \operatorname{Re}(z)>0\}$. We consider the following cases:

Case A. $c=\gamma=0$ and $\mathcal{S}$ is a driftless subordinator other than a compound Poisson process or $\mathcal{S}$ is a compound Poisson process such that $\mathbb{E}[\mathcal{X}(1)]>0$,

Case B. $c>0, \gamma=0$ and $\mathcal{S}$ is a driftless subordinator,

Case C. Any other case, except when $c=\gamma=0$ and $\mathcal{S}$ is a compound Poisson processes with $\mathbb{E}[\mathcal{X}(1)] \leq 0$. In this case we also assume that $\int_{0+}^{\infty}\left(x^{2} \wedge x\right) \nu_{\mathcal{S}}(d x)<\infty$.

Remark 1 Assumption $\int_{0+}^{\infty}\left(x^{2} \wedge x\right) \nu_{\mathcal{S}}(d x)<\infty$ is true, for instance, when $\int_{0+}^{\infty} x \nu_{\mathcal{S}}(d x)<\infty$.

The following result from Lewis and Mordecki [2008] holds for the roots of the equation $\Psi_{\mathcal{X}}(r)-q=0$, which we call generalized Cramér-Lundberg equation:

Lemma 1 Let $q \geq 0$ and assume $\mathbb{E}[\mathcal{X}(1)]>0$ when $q=0$. Then:

a) In case $A$, the equation $\Psi_{\mathcal{X}}(r)-q=0$ has $m$ roots in $\mathbb{C}_{++}$,

b) In cases $B$ and $C$, the equation $\Psi_{\mathcal{X}}(r)-q=0$ has $m+1$ roots in $\mathbb{C}_{++}$.

In all the cases above, there is exactly one real root $\beta_{1}(q)$ in the interval $\left(0, \alpha_{1}\right)$, and it satisfies $\lim _{q \downarrow 0} \beta_{1}(q)=$ 0. When $q=0, \beta_{1}(0)=0$ is a simple root of $\Psi_{\mathcal{X}}(r)=0$ in all cases $A, B$ and $C$. 
Let us assume that the equation $\Psi_{\mathcal{X}}(r)-q=0$ has $R$ different roots in $\mathbb{C}_{++}$, denoted by $\beta_{1}(q), \ldots, \beta_{R}(q)$, with respectively multiplicities $k_{1}, k_{2}, \ldots, k_{R}$, where $\sum_{j=1}^{R} k_{j}=m+1-1_{\{\text {case } A\}}$, and $1_{\{\text {case } A\}}=1$ in case $\mathrm{A}$ and $1_{\{\text {case } A\}}=0$ in the other cases. We let $\beta_{1}(q)$ be the real root such that $\beta_{1}(q) \in\left[0, \alpha_{1}\right)$, hence $k_{1}=1$.

The case when $q=0$ is taken in the limiting sense.

When $\mathbb{E}[\mathcal{X}(1)] \leq 0$, we have $\mathbb{P}\left[I_{\infty}=-\infty\right]=1$, hence we have the following condition:

Condition 1 For $q=0$, we assume that $\mathbb{E}[\mathcal{X}(1)]>0$.

For $a=0,1, \ldots, m+1$, we define the linear operator $\mathcal{T}_{s, a}$ by the expression

$$
\mathcal{T}_{s, a} f(u)=\int_{u}^{\infty}(y-u)^{a} e^{-s(y-u)} f(y) d y
$$

for all measurable, nonnegative functions $f$ and complex numbers $s$ such that the integral above exists and is finite. If $\nu$ is a measure such that $\int_{u}^{\infty}(y-u)^{a} e^{-s(y-u)} \nu(d y)$ exists, we define for $a=0,1, \ldots, m+1$,

$$
\mathcal{T}_{s, a} \nu(u)=\int_{u}^{\infty}(y-u)^{a} e^{-s(y-u)} \nu(d y)
$$

and denote the Laplace transforms of these two operators by $\widehat{\mathcal{T}}_{s, a} f$ and $\widehat{\mathcal{T}}_{s, a} \nu$. When $a=0$, we obtain the Dickson-Hipp operator $T_{s} f$ defined in Dickson and Hipp [2001] and write $\mathcal{T}_{s} f(u)=\int_{u}^{\infty} e^{-s(y-u)} f(y) d y$, with the corresponding modification when $f$ is replaced by a measure $\nu$. We shall use the following elementary properties and lemma:

$$
\widehat{T}_{s} f(r)=\frac{\widehat{f}(r)-\widehat{f}(s)}{s-r}, \quad \widehat{T}_{s} \nu(r)=\frac{\int_{0+}^{\infty}\left(e^{-r x}-e^{-s x}\right) \nu(d x)}{s-r}
$$

Lemma 2 Let $f$ be a function (or a measure) such that $\mathcal{T}_{s, k} f(u)$ exists for every $s \in \mathbb{C}_{++}, k \in \mathbb{N} \cup\{0\}$ and $u>0$. For each $r \in \mathbb{C}_{+}, s \in \mathbb{C}_{++}$and $k \in \mathbb{N} \cup\{0\}$ there holds $\frac{\partial^{k}}{\partial s^{k}} \widehat{T}_{s} f(r)=(-1)^{k} \widehat{\mathcal{T}}_{s, k} f(r)$.

The following result follows from Theorem 6.16 in Kyprianou [2006].

Lemma 3 Let $S_{e_{q}}$ be the positive Wiener-Hopf factor of a Lévy process, other than a compound Poisson process, and denote by $\kappa$ the joint Laplace exponent for the bivariate subordinator representing the ascending ladder process $\left(\mathbb{L}^{-1}, \mathbb{H}\right)$, and by $\Lambda(d x, d y)$ its bivariate Lévy measure. Then there exist $b \geq 0$ such that, for $r \geq 0$ and $q>0$, it holds

$$
b r+\int_{0+}^{\infty}\left(1-e^{-r y}\right) \int_{0+}^{\infty} e^{-q x} \Lambda(d x, d y)=\kappa(q, r)-\kappa(q, 0)
$$


and

$$
\mathbb{E}\left[e^{-r S_{e_{q}}}\right]=E\left[e^{-r \mathbb{H}_{q}\left(e_{\kappa(q, 0)}\right)}\right]=\frac{\kappa(q, 0)}{\kappa(q, r)} .
$$

Here $e_{\kappa(q, 0)}$ is an exponential random variable with mean $1 / \kappa(q, 0)$, independent of the Lévy process. It also holds

$$
q-\Psi_{\mathcal{X}}(r)=\kappa(q,-i r) \widehat{\kappa}(q, i r)
$$

where $\widehat{\kappa}$ is the joint Laplace exponent for the bivariate subordinator representing the descending ladder process $\left(\mathbb{L}^{-1}, \widehat{\mathbb{H}}\right)$.

In order to simplify our notations, we define the following constants:

$$
\begin{gathered}
E(j, a, q)=\left(\begin{array}{c}
k_{j}-1 \\
a
\end{array}\right) \frac{(-1)^{1-k_{j}+a}}{\left(k_{j}-1\right) !} \frac{\partial^{k_{j}-1-a}}{\partial s^{k_{j}-1-a}}\left[\frac{\prod_{l=1}^{N}\left(\alpha_{l}-s\right)^{n_{l}}\left(\beta_{j}(q)-s\right)^{k_{j}}}{\prod_{l=1}^{R}\left(\beta_{l}(q)-s\right)^{k_{l}}}\right]_{s=\beta_{j}(q)}, \\
E_{*}(j, a, q)=\left(\begin{array}{c}
k_{j}-1 \\
a
\end{array}\right) \frac{(-1)^{1-k_{j}+a}}{\left(k_{j}-1\right) !} \frac{\partial^{k_{j}-1-a}}{\partial s^{k_{j}-1-a}}\left[\frac{\prod_{l=1}^{N}\left(\alpha_{l}-s\right)^{n_{l}}\left(\beta_{j}(q)-s\right)^{k_{j}}}{\prod_{l=1}^{R}\left(\beta_{l}(q)-s\right)^{k_{l}}} s\right]_{s=\beta_{j}(q)},
\end{gathered}
$$

for each $j=1,2, \ldots, R$. The constants $E(j, 0, q)$ and $E(j, a, q)$ for $a>0$ correspond, respectively, to those given in expressions (2.4) and (2.5) in Lewis and Mordecki [2008].

We define the functions

$$
\ell_{q}(u)=\sum_{j=1}^{R} \sum_{a=0}^{k_{j}-1} E(j, a, q) \mathcal{T}_{\beta_{j}(q), a} \nu_{\mathcal{S}}(u), \quad \mathcal{L}_{q}(u)=\sum_{j=1}^{R} \sum_{a=0}^{k_{j}-1} E_{*}(j, a, q) \mathcal{T}_{\beta_{j}(q), a} \overline{\mathcal{V}}_{\mathcal{S}}(u), \quad q \geq 0,
$$

and the measure

$$
\chi_{q, \mathcal{S}}(d x)= \begin{cases}\nu_{\mathcal{S}}(d x)+\ell_{q}(x) d x & \text { in case } \mathrm{A}, \\ \ell_{q}(x) d x & \text { in case } \mathrm{B}, \\ {\left[\overline{\mathcal{V}}_{\mathcal{S}}(x)-\mathcal{L}_{q}(x)\right] d x} & \text { in case } \mathrm{C} .\end{cases}
$$

\section{Main results}

In this section we obtain an explicit expression for the probability density of the negative Wiener-Hopf factor $I_{e_{q}}$ of the process $\mathcal{X}$ defined in (2.1). The results presented for $q=0$ are all under the assumption that Condition 1 holds. 
For $a>0$ let $\mathcal{E}_{a}(x)$ denote the exponential density $\mathcal{E}_{a}(x)=a e^{-a x}, x>0$ and define, for $q \geq 0$, the function $\widehat{W}_{q}$ by

$$
\widehat{W}_{q}(r)=\frac{\kappa(q,-r)}{\left[q-\Psi_{\mathcal{X}}(r)\right]}, \quad r \geq 0
$$

where $\kappa$ is given in Lemma3, By Theorem 2.2 in Lewis and Mordecki [2008], we know that

$$
\kappa(q, r)=\frac{\prod_{j=1}^{R}\left(\beta_{j}(q)+r\right)^{k_{j}}}{\prod_{l=1}^{N}\left(\alpha_{l}+r\right)^{n_{l}}}
$$

so $\kappa(q, 0)=\frac{\prod_{j=1}^{R} \beta_{j}^{k_{j}}(q)}{\prod_{l=1}^{N} \alpha_{l}^{n l}(q)}$. Since $\beta_{1}(0)=0$, it follows that $\kappa(0,0)=0$. Hence, using that from L'Hôpital's rule $\lim _{\beta_{1}(q) \rightarrow 0} \frac{\Psi_{\mathcal{X}}\left(\beta_{1}(q)\right)}{\left.\beta_{1}(q)\right)}=\mathbb{E}(\mathcal{X}(1))$, we obtain

$$
\lim _{q \downarrow 0} \frac{q}{\kappa(q, 0)}=\lim _{q \downarrow 0} \frac{q}{\beta_{1}(q)} \frac{\prod_{l=1}^{N} \alpha_{l}^{n_{l}}}{\prod_{j=2}^{R} \beta_{j}^{k_{j}}(q)}=\mathbb{E}[\mathcal{X}(1)] \frac{\prod_{l=1}^{N} \alpha_{l}^{n_{l}}}{\prod_{j=2}^{R} \beta_{j}^{k_{j}}(0)}
$$

We define for $q>0, a(q)=q / \kappa(q, 0)$ and $a(0)=\lim _{q \downarrow 0} a(q)$.

Hence, we have the following result.

Lemma 4 The Laplace transforms of $-I_{e_{q}}$ for $q>0$ and $-I_{\infty}$ satisfy the following equalities for $r \geq 0$ :

$$
\mathbb{E}\left[e^{-r\left[-I_{e_{q}}\right]}\right]=a(q) \widehat{W}_{q}(r) \quad \text { and } \quad \mathbb{E}\left[e^{-r\left[-I_{\infty}\right]}\right]=a(0) \widehat{W}_{0}(r)
$$

Hence, for $q \geq 0, a(q) W_{q}$ is the density of $-I_{e_{q}}$.

Proof. Using (1.1), Lemma 3 and the relation $\psi_{\mathcal{X}}(s)=-\Psi_{\mathcal{X}}(i s)$, we get

$$
\mathbb{E}\left[e^{i s I_{e_{q}}}\right]=\frac{q}{q-\Psi_{\mathcal{X}}(i s)}\left(\frac{\kappa(q,-i s)}{\kappa(q, 0)}\right) .
$$

The function on the right-hand side can be analytically extended to negative part of the imaginary axis. Hence the result follows taking $s=-i r$ for $r \geq 0$.

The case for $q=0$ follows by taking limits when $q \downarrow 0$.

In the following result we invert $a(q) \widehat{W}_{q}$.

\section{Theorem 1}


a) The function $\widehat{W}_{q}$ satisfies the equalities

$$
a(q) \widehat{W}_{q}(r)= \begin{cases}\frac{\widehat{\mathcal{E}}_{a(q)}(r)}{1-\left(1-\widehat{\mathcal{E}}_{a(q)}(r)\right)\left(1-\hat{\bar{\chi}}_{q, \mathcal{S}}(r)\right)}, & \text { in cases } A \text { and } C \text { with } \gamma=0, \\ \frac{\frac{a(q)}{c}}{1-\frac{1}{c} \widehat{\chi}_{q, \mathcal{S}}(r)}, & \text { in case } B \\ \frac{\widehat{\mathcal{E}}_{a(q) \gamma}-2(r)}{1+\gamma^{-2}\left(1-\widehat{\mathcal{E}}_{a(q) \gamma-2}(r)\right) \hat{\bar{\chi}}_{q, \mathcal{S}}(r)}, & \text { in case } C \text { with } \gamma>0 .\end{cases}
$$

b) For $q \geq 0$ and $u \geq 0$ the negative Wiener-Hopf factor $I_{e_{q}}$ has a generalized density function given by:

$$
\begin{aligned}
& a(q) W_{q}(u) \\
& = \begin{cases}\mathcal{E}_{a(q)} * \sum_{n=0}^{\infty} \sum_{k=0}^{n}\left(\begin{array}{l}
n \\
k
\end{array}\right)(-1)^{k}\left(\bar{\chi}_{q, \mathcal{S}}+\mathcal{E}_{a(q)}-\mathcal{E}_{a(q)} * \bar{\chi}_{q, \mathcal{S}}\right)^{* k}(u) & \text { in cases A and } C \text { with } \gamma=0, \\
\frac{a(q)}{c} \delta_{0}(u)+\frac{a(q)}{c} \sum_{n=1}^{\infty}\left(\frac{1}{c}\right)^{n} \chi_{q, \mathcal{S}}^{* n}(u), & \text { in case B, } \\
\mathcal{E}_{a(q) \gamma^{-2}} * \sum_{n=0}^{\infty}\left(-\frac{1}{\gamma^{2}}\right)^{n}\left(\bar{\chi}_{q, \mathcal{S}}-\mathcal{E}_{a(q) \gamma^{-2}} * \bar{\chi}_{q, \mathcal{S}}\right)^{* n}(u) & \text { in case } C \text { with } \gamma>0,\end{cases}
\end{aligned}
$$

where $\delta_{0}$ is Dirac's delta function.

In order to prove Theorem 1 we need the following lemma. Its proof is technical and lengthly and is deferred to section 6 .

Lemma 5 For $q \geq 0$ we have:

$$
\left[q-\Psi_{\mathcal{X}}(r)\right](\kappa(q,-r))^{-1}= \begin{cases}a(q)+G_{\mathcal{S}}(r)+\widehat{\ell}_{q}(0)-\widehat{\ell}_{q}(r), & \text { in Case A, } \\ a(q)+\widehat{\ell}_{q}(0)-\widehat{\ell}_{q}(r), & \text { in Case B, } \\ a(q)+\gamma^{2} r-\frac{\Psi_{\mathcal{S}}(r)}{r}-\left[\widehat{\mathcal{L}}_{q}(0)-\widehat{\mathcal{L}}_{q}(r)\right], & \text { in Case C. }\end{cases}
$$

Proof of Theorem 1. Clearly, part b) follows inverting (3.5). To prove (3.5) we assume $q>0$. The case $q=0$ follows by letting $q \downarrow 0$.

From (3.3), 2.7), 3.1), 3.6) and the definition of $a(q)$ we obtain

$$
\mathbb{E}\left[e^{-r\left[-I_{e_{q}}\right]}\right]=a(q) \widehat{W}_{q}(r)=\frac{a(q)}{a(q)+\int_{0+}^{\infty}\left(1-e^{-r x}\right) \chi_{q, \mathcal{S}}(d x)}=\frac{q}{q+\int_{0+}^{\infty}\left(1-e^{-r x}\right) \kappa(q, 0) \chi_{q, \mathcal{S}}(d x)}
$$

To obtain (3.5) in case A, we use

$$
\widehat{\mathcal{E}}_{a(q)}(r)=\frac{a(q)}{a(q)+r}
$$


and apply Fubini's Theorem to $\int_{0+}^{\infty}\left(1-e^{-r x}\right) \chi_{q, \mathcal{S}}(d x)$. This yields,

$$
a(q) \widehat{W}_{q}(r)=\frac{a(q)}{a(q)+\int_{0+}^{\infty}\left(1-e^{-r x}\right) \chi_{q, \mathcal{S}}(d x)}=\frac{a(q)}{a(q)+r \widehat{\bar{\chi}}_{q, \mathcal{S}}(r)}=\frac{\widehat{\mathcal{E}}_{a(q)}(r)}{1-\left(1-\widehat{\mathcal{E}}_{a(q)}(r)\right)\left(1-\widehat{\bar{\chi}}_{q, \mathcal{S}}(r)\right)}
$$

Hence (3.5) follows in this case. In case B, (3.1) and (3.6) we have

$$
a(q) \widehat{W}_{q}(r)=\frac{a(q)}{a(q)+\widehat{\ell}_{q}(0)-\widehat{\ell}_{q}(r)}=\frac{\frac{a(q)}{a(q)+\widehat{\ell}_{q}(0)}}{1-\frac{1}{a(q)+\widehat{\ell}_{q}(0)} \widehat{\ell}_{q}(r)} .
$$

Due to (6.13) we have $a(q)+\widehat{\ell}_{q}(0)=c$, and from 2.7) it follows that $\widehat{\ell}_{q}(r)=\widehat{\chi}_{q, \mathcal{S}}(r)$. Substituting these two equalities into (3.9) and using (3.3) gives (3.5).

We now deal with case C. Using (3.1) and (3.6), we obtain

$$
a(q) \widehat{W}_{q}(r)=\frac{a(q)}{a(q)+\gamma^{2} r-\frac{\Psi_{\mathcal{S}}(r)}{r}-\left[\widehat{\mathcal{L}}_{q}(0)-\widehat{\mathcal{L}}_{q}(r)\right]} .
$$

Now we apply Fubini's theorem to $\Psi_{\mathcal{S}}(r) / r$ to obtain, for $\gamma>0$ :

$$
a(q) \widehat{W}_{q}(r)=\frac{\frac{a(q) \gamma^{-2}}{a(q) \gamma^{-2}+r}}{1+\gamma^{-2} \frac{r}{a(q) \gamma^{-2}+r} \widehat{\bar{\chi}}_{q, \mathcal{S}}(r)}=\frac{\widehat{\mathcal{E}}_{a(q) \gamma^{-2}(r)}}{1+\gamma^{-2}\left(1-\widehat{\mathcal{E}}_{a(q) \gamma^{-2}(r)}\right) \widehat{\bar{\chi}}_{q, \mathcal{S}}(r)},
$$

where we have used (3.8) with $a(q)$ replaced by $a(q) \gamma^{-2}$. When $\gamma=0$ it holds,

$$
a(q) \widehat{W}_{q}(r)=\frac{\frac{a(q)}{a(q)+r}}{1+\frac{r}{a(q)+r} \widehat{\bar{\chi}}_{q, \mathcal{S}}(r)}=\frac{\widehat{\mathcal{E}}_{a(q)(r)}}{1+\left(1-\widehat{\mathcal{E}}_{a(q)(r)}\right) \widehat{\bar{\chi}}_{q, \mathcal{S}}(r)},
$$

and we obtain (3.5) using (3.3).

Lemma 6 For all $q \geq 0$ the measure $\kappa(q, 0) \chi_{q, \mathcal{S}}$ is the Lévy measure of $-I_{e_{q}}$.

Proof. The non-negative random variable $-I_{e_{q}}$ is infinitely divisible, with Laplace transform

$$
\mathbb{E}\left[e^{-r\left(-I_{e_{q}}\right)}\right]=\exp \left\{-\int_{0+}^{\infty}\left(1-e^{-r x}\right) \nu_{q}(d x)\right\}
$$

where the measure

$$
\nu_{q}(d x)=\int_{0}^{\infty} t^{-1} e^{-q t} \mathbb{P}\left[-I_{t} \in d x\right] d t
$$

is the Lévy measure of $-I_{e_{q}}$ (see e.g. Lema 6.17 in Kyprianou [2006]). On the other hand, from the formula for Frullani's integral, we have for $\alpha, \beta>0$ and $z \leq 0$,

$$
\left(\frac{\alpha}{\alpha-z}\right)^{\beta}=\exp \left\{-\int_{0}^{\infty}\left(1-e^{z t}\right) \beta t^{-1} e^{-\alpha t} d t\right\}
$$


Let $\mathcal{N}_{q}$ denote the subordinator with Lévy measure $\kappa(q, 0) \chi_{q, \mathcal{S}}$, and denote its Laplace exponent by $\Psi_{q}$. From (3.7) we have $\mathbb{E}\left[e^{-r\left(-I_{e_{q}}\right)}\right]=\frac{q}{q+\Psi_{q}(r)}$, hence using (3.12) with $\alpha=q, \beta=1$ and $z=-\Psi_{q}(r)$ we obtain

$$
\mathbb{E}\left[e^{-r\left(-I_{e_{q}}\right)}\right]=\exp \left\{-\int_{0}^{\infty}\left(1-e^{-t \Psi_{q}(r)}\right) t^{-1} e^{-q t} d t\right\} .
$$

Since $1-e^{-t \Psi_{q}(r)}=\int_{0}^{\infty}\left(1-e^{-r x}\right) \mathbb{P}\left[\mathcal{N}_{q}(t) \in d x\right]$, setting

$$
\pi(d x)=\int_{0}^{\infty} t^{-1} e^{-q t} \mathbb{P}\left[\mathcal{N}_{q}(t) \in d x\right] d t
$$

and using Fubini's theorem in 3.13 it follows that

$$
\mathbb{E}\left[e^{-r\left(-I_{e_{q}}\right)}\right]=\exp \left\{-\int_{0}^{\infty}\left(1-e^{-r x}\right) \pi(d x)\right\} .
$$

Now from (3.10) we deduce that

$$
\pi=\nu_{q}
$$

Using (3.14) and (3.11) we obtain the result.

Remark 2 Since $-I_{e_{q}}=\widehat{H}\left(e_{\widehat{\kappa}(q, 0)}\right)$ in distribution [Kvprianou, 2006, Theorem 6.16], it follows that the measure $\kappa(q, 0) \chi_{q, \mathcal{S}}$ is also the Lévy measure of the descending ladder-height process $\widehat{H}$ corresponding to $\mathcal{X}(t)$, killed at the uniform rate $\widehat{\kappa}(q, 0)$.

\section{Asymptotic behavior of the negative Wiener-Hopf factor}

For $u>0$ we denote $F_{I_{e_{q}}}(-u)=\mathbb{P}\left[I_{e_{q}}<-u\right]$

We obtain asymptotic expressions for $F_{I_{e_{q}}}(-u)$ when $u \rightarrow \infty$. For this, we use the following technical result.

Lemma 7 The equality

$$
r \widehat{F}_{I_{e_{q}}}(r)=\frac{\sum_{j=1}^{m+2} A_{j}^{\prime} r^{j}+\Psi_{\mathcal{S}}(r) \prod_{l=1}^{N} \alpha_{l}^{n_{l}}+\sum_{j=1}^{m} A_{j} r^{j} \Psi_{\mathcal{S}}(r)-\widehat{\kappa}(q, 0) \sum_{j=1}^{m+1} B_{j} r^{j}}{q \prod_{l=1}^{N} \alpha_{l}^{n_{l}}+\sum_{j=1}^{m+2} A_{j}^{\prime} r^{j}+\Psi_{\mathcal{S}}(r) \prod_{l=1}^{N} \alpha_{l}^{n_{l}}+\sum_{j=1}^{m} A_{j} r^{j} \Psi_{\mathcal{S}}(r)}
$$

holds for some constants $A_{j}, j=1, \ldots, m, B_{k}, k=1, \ldots, m+1$ and $A_{l}^{\prime}, l=1, \ldots, m+2$.

Proof. Using that $r \widehat{F}_{I_{e_{q}}}(r)=1-\widehat{f}_{-I_{e_{q}}}(r)$, where $\widehat{f}_{-I_{e_{q}}}(r)$ denotes the Laplace transform of the density of $-I_{e_{q}}$, we obtain as in (2.5)

$$
r \widehat{F}_{I_{e_{q}}}(r)=\frac{\widehat{\kappa}(q, r)-\widehat{\kappa}(q, 0)}{\widehat{\kappa}(q, r)}
$$


On the other hand from (2.6) and (3.2) it follows

$$
\widehat{\kappa}(q, r)=\prod_{l=1}^{N}\left(\alpha_{l}-r\right)^{n_{l}} \frac{q-\Psi_{\mathcal{X}}(r)}{\prod_{j=1}^{R}\left(\beta_{j}(q)-r\right)^{k_{j}}} .
$$

Since

$$
q-\Psi_{\mathcal{X}}(r)=q-c r-\gamma^{2} r^{2}-\lambda_{1}\left(\frac{Q(-r)}{\prod_{j=1}^{N}\left(\alpha_{j}-r\right)^{n_{j}}}-1\right)+\Psi_{\mathcal{S}}(r)
$$

and $\lambda_{1}\left(\frac{Q(-r)}{\prod_{j=1}^{N}\left(\alpha_{j}-r\right)^{n_{j}}}-1\right)$ can be written as the quotient of some polynomial $\mathcal{P}_{1}$ with degree $m$ and $\prod_{j=1}^{N}\left(\alpha_{j}-r\right)^{n_{j}}$, which is also a polynomial of degree $m$, we have

$$
q-\Psi_{\mathcal{X}}(r)=q-c r-\gamma^{2} r^{2}-\frac{\mathcal{P}_{1}(r)}{\prod_{j=1}^{N}\left(\alpha_{j}-r\right)^{n_{j}}}+\Psi_{\mathcal{S}}(r)
$$

Since $\frac{Q(0)}{\prod_{j=1}^{N} \alpha_{j}^{n_{j}}}=1$, we obtain that $\mathcal{P}_{1}$ is a polynomial with constant term 0 . Using $\prod_{j=1}^{N}\left(\alpha_{j}-r\right)^{n_{j}}=$ $\sum_{j=0}^{m} A_{j} r^{j}$, it follows that

$$
\begin{aligned}
\prod_{j=1}^{N}\left(\alpha_{j}-r\right)^{n_{j}}\left(q-\Psi_{\mathcal{X}}(r)\right) & =q A_{0}+q \sum_{j=1}^{m} A_{j} r^{j}-c \sum_{j=0}^{m} A_{j} r^{j+1}-\gamma^{2} \sum_{j=0}^{m} A_{j} r^{j+2} \\
& -\mathcal{P}_{1}(r)+A_{0} \Psi_{\mathcal{S}}(r)+\sum_{j=1}^{m} A_{j} r^{j} \Psi_{\mathcal{S}}(r) \\
& =q A_{0}+\sum_{j=1}^{m+2} A_{j}^{\prime} r^{j}+A_{0} \Psi_{\mathcal{S}}(r)+\sum_{j=1}^{m} A_{j} r^{j} \Psi_{\mathcal{S}}(r) .
\end{aligned}
$$

Evaluating $\prod_{j=1}^{N}\left(\alpha_{j}-r\right)^{n_{j}}$ at $r=0$ we obtain $A_{0}=\prod_{l=1}^{N} \alpha_{l}^{n_{l}}$. Moreover, setting $r=0$ in (4.3) gives

$$
q \prod_{l=1}^{N} \alpha_{l}^{n_{l}}=\widehat{\kappa}(q, 0) \prod_{j=1}^{R} \beta_{j}^{k_{j}}(q) .
$$

Hence, using that $\prod_{j=1}^{R}\left(\beta_{j}(q)-r\right)^{k_{j}}$ can be expressed as $\sum_{j=0}^{m+1} B_{j} r^{j}$ and substituting (4.6) and (4.5) into 
(4.2), it follows that

$$
r \widehat{F}_{I_{e_{q}}}(r)=\frac{\sum_{j=1}^{m+2} A_{j}^{\prime} r^{j}+\Psi_{\mathcal{S}}(r) \prod_{l=1}^{N} \alpha_{l}^{n_{l}}+\sum_{j=1}^{m} A_{j} r^{j} \Psi_{\mathcal{S}}(r)-\widehat{\kappa}(q, 0) \sum_{j=1}^{m+1} B_{j} r^{j}}{q \prod_{l=1}^{N} \alpha_{l}^{n_{l}}+\sum_{j=1}^{m+2} A_{j}^{\prime} r^{j}+\Psi_{\mathcal{S}}(r) \prod_{l=1}^{N} \alpha_{l}^{n_{l}}+\sum_{j=1}^{m} A_{j} r^{j} \Psi_{\mathcal{S}}(r)} .
$$

In what follows we write $f \approx c g$ for any two nonnegative functions $f$ and $g$ on $[0, \infty)$ such that $\lim _{u \rightarrow \infty} \frac{f(u)}{g(u)}=$ $c$, with $c \neq 0$. Now we can derive the first asymptotic expression for $F_{I_{e_{q}}}$.

Proposition 1 If $r^{-\xi} \Psi_{\mathcal{S}}(r) \rightarrow D$ as $r \downarrow 0$ for some $\xi \in(0,1)$ and some positive constant $D$, then

$$
F_{I_{e_{q}}}(-u) \approx \frac{D}{q \Gamma(1-\xi)} u^{-\xi}, \quad u \rightarrow \infty
$$

Proof. Due to Theorem 4 in Feller [1971], page 446, we only need to prove that

$$
r^{-\xi+1} \widehat{F}_{I_{e q}}(r) \rightarrow D q^{-1}
$$

From (4.1) we have

$$
r^{-\xi+1} \widehat{F}_{I_{e_{q}}}(r)=\frac{\sum_{j=1}^{m+2} A_{j}^{\prime} r^{j-\xi}+r^{-\xi} \Psi_{\mathcal{S}}(r) \prod_{l=1}^{N} \xi_{l}^{n_{l}}+\sum_{j=1}^{m} A_{j} r^{j-\xi} \Psi_{\mathcal{S}}(r)-\widehat{\kappa}(q, 0) \sum_{j=1}^{m+1} B_{j} r^{j-\xi}}{q \prod_{l=1}^{N} \xi_{l}^{n_{l}}+\sum_{j=1}^{m+2} A_{j}^{\prime} r^{j}+\Psi_{\mathcal{S}}(r) \prod_{l=1}^{N} \xi_{l}^{n_{l}}+\sum_{j=1}^{m} A_{j} r^{j} \Psi_{\mathcal{S}}(r)} .
$$

Since all the polynomial terms in the numerator have no constant term and $\xi \in(0,1)$, we have $j-\xi>0$, hence letting $r \downarrow 0$ and using the hypothesis on $r^{-\xi} \Psi_{\mathcal{S}}(r)$, we obtain (4.7).

Let us denote by $\bar{\chi}_{q, \mathcal{S}}$ the tail of the Lévy measure $\chi_{q, \mathcal{S}}$.

Proposition 2 In case $B$, if $1-\frac{\bar{\chi}_{q, \mathcal{S}}(u)}{\widehat{\chi}_{q, \mathcal{S}}(0)}$ is a subexponential distribution, then $F_{I_{e_{q}}}(-u) \approx q^{-1} \kappa(q, 0) \bar{\chi}_{q, \mathcal{S}}(u)$.

Proof. First we note that from 6.13) we get $c=a(q)+\widehat{\ell}_{q}(0)$, hence $a(q)=c-\widehat{\chi}_{q, \mathcal{S}}(0)$, and

$$
\widehat{F}_{I_{e_{q}}}(r)=\frac{1}{r}\left(\frac{\frac{1}{c} \widehat{\chi}_{q, \mathcal{S}}(0)-\frac{1}{c} \widehat{\chi}_{q, \mathcal{S}}(r)}{1-\frac{1}{c} \widehat{\chi}_{q, \mathcal{S}}(r)}\right)=\frac{\frac{1}{c} \widehat{\bar{\chi}}_{q, \mathcal{S}}(r)}{1-\frac{1}{c} \widehat{\chi}_{q, \mathcal{S}}(r)},
$$

where in the last equality we used that for a probability density $f$ with tail $\bar{F}$, we have $\widehat{\bar{F}}(r)=r^{-1}(1-\widehat{f}(r))$. It follows that

$$
F_{I_{e_{q}}}(-u)=\frac{1}{c} \bar{\chi}_{q, \mathcal{S}} * \sum_{n=0}^{\infty}\left(\frac{1}{c}\right)^{n} \bar{\chi}_{q, \mathcal{S}}^{* n}(u) .
$$


Now we set $p=\frac{\widehat{\chi}_{q, \mathcal{S}}(0)}{a(q)+\widehat{\chi}_{q, \mathcal{S}}(0)}$. Then $p \in(0,1)$ and from (6.13) it follows that $p=\frac{\widehat{\chi}_{q, \mathcal{S}}(0)}{c}$. Using (4.9) we get

$$
F_{I_{e_{q}}}(-u)=\frac{1}{c} \bar{\chi}_{q, \mathcal{S}} * \sum_{n=0}^{\infty}\left(\frac{1}{c}\right)^{n} \chi_{q, \mathcal{S}}^{* n}(u)=p \frac{\bar{\chi}_{q, \mathcal{S}}}{\widehat{\chi}_{q, \mathcal{S}}(0)} * \sum_{n=0}^{\infty} p^{n}\left(\frac{\chi_{q, \mathcal{S}}}{\widehat{\chi}_{q, \mathcal{S}}(0)}\right)^{* n}(u) .
$$

Let us define the probability distribution $H(u)=(1-p) \int_{0}^{u} \sum_{n=0}^{\infty} p^{n}\left(\frac{\chi_{q, \mathcal{S}}}{\widehat{\chi}_{q, \mathcal{S}}(0)}\right)^{* n}(x) d x$, and denote its density by $h$. Since $\bar{\chi}_{q, \mathcal{S}}(u) / \widehat{\chi}_{q, \mathcal{S}}(0)$ is the tail of a proper distribution, due to Corollary 3 in Embrechts et al. [1979] and the assumption that $1-\bar{\chi}_{q, \mathcal{S}} / \widehat{\chi}_{q, \mathcal{S}}(0)$ is subexponential, we obtain

$$
\bar{H}(u) \approx \frac{p}{1-p} \frac{\bar{\chi}_{q, \mathcal{S}}(u)}{\widehat{\chi}_{q, \mathcal{S}}(0)}
$$

hence $H$ is a subexponential distribution. From Lemma 2.5.2 in Rolski et al. [1999] and 4.10), we have

$$
\lim _{u \rightarrow \infty} \frac{F_{I_{e_{q}}}(-u)}{\bar{\chi}_{q, \mathcal{S}}(u) / \widehat{\chi}_{q, \mathcal{S}}(0)}=\lim _{u \rightarrow \infty} \frac{\frac{p}{1-p} \frac{\bar{\chi}_{q, \mathcal{S}}}{\widehat{\chi}_{q, \mathcal{S}}(0)} * h(u)}{\bar{\chi}_{q, \mathcal{S}}(u) / \widehat{\chi}_{q, \mathcal{S}}(0)}=\frac{p}{1-p}=\widehat{\chi}_{q, \mathcal{S}}(0) q^{-1} \kappa(q, 0),
$$

which implies the result.

Let us define $\Pi(x)=\frac{\int_{1}^{x} \chi_{q, \mathcal{S}}(d y)}{\int_{1}^{\infty} \chi_{q, \mathcal{S}}(d y)}$ for $x>1$. We recall that a probability distribution $F$ is a subexponential distribution if $\overline{F^{* 2}}(x) \approx 2 \bar{F}(x)$ as $x \rightarrow \infty$.

Proposition 3 Suppose that $\Pi$ is a subexponential distribution and set $\overline{\nu_{q}}(u)=\int_{u}^{\infty} \nu_{q}(d y)$ where $\nu_{q}$ is the Lévy measure of $-I_{e_{q}}$. Then the random variable $-I_{e_{q}}$ has a subexponential distribution, and

$$
F_{I_{e_{q}}}(-u) \approx \overline{\nu_{q}}(u), u \rightarrow \infty
$$

Proof. The assertion that $-I_{e_{q}}$ has a subexponential distribution and that 4.11) holds, follow from (3.15) and Theorem 1 in Embrechts et al. [1979].

\section{Examples}

In this section we apply the results from the previous section to several particular examples in which we obtain simple asymptotic expressions for the negative Wiener-Hopf factor $F_{I_{e_{q}}}$. For simplicity, in cases A and $\mathrm{C}$ we will assume that the roots of $\Psi_{\mathcal{X}}(r)-q=0$ in $\mathbb{C}_{++}$are all different. This assumption holds e.g. when the density $f_{1}$ is a convex combination of exponential densities.

Example 1 (case A). We take $\Psi_{\mathcal{S}}(r)=r^{\xi}$, for $\xi \in(0,1)$, hence $\mathcal{S}$ is an $\xi$-stable subordinator and the assumptions on Proposition 1 hold. Hence,

$$
F_{I_{e_{q}}}(-u) \approx \frac{1}{\Gamma(1-\xi)} u^{-\xi} \text { as } u \rightarrow \infty
$$


Example 2 (Case B). 1. Let us suppose that $G_{\mathcal{S}}(r)=\lambda_{2} \widehat{f_{2}}(r)-\lambda_{2}$, i.e. $\mathcal{S}$ is a compound Poisson process with Lévy measure $\lambda_{2} f_{2}(x)$ with $\lambda_{2}>0$. In this case the resulting Lévy risk process is the classical two-sided jumps risk process. Therefore

$$
\chi_{q, \mathcal{S}}(u)=\lambda_{2} \sum_{j=1}^{R} \sum_{a=0}^{k_{j}-1} E(j, a, q) \int_{u}^{\infty}(y-u)^{a} e^{-\beta_{j}(q)(y-u)} f_{2}(y) d y
$$

When $k_{j}=1$ for all $j=1,2, \ldots, R$, and $f_{2}$ is a mixture of exponential densities, the above expression can be easily calculated. Let us consider the particular case when $f_{1}(x)=q e^{-q x}, x>0, \lambda_{2}=1$ and $f_{2}(x)=p e^{-p x}, x>0$. In this case the generalized Lundberg equation $\Psi_{\mathcal{X}}(r)-q=0$ has two real roots $\beta_{1}(q)$ and $\beta_{2}(q)$ such that $0<\beta_{1}(q)<q<\beta_{2}(q)$. Hence

$$
\chi_{q, \mathcal{S}}(u)=\left(\frac{q-\beta_{1}(q)}{\beta_{2}(q)-\beta_{1}(q)} \frac{1}{\beta_{1}(q)+p}+\frac{\beta_{2}(q)-q}{\beta_{2}(q)-\beta_{1}(q)} \frac{1}{\beta_{2}(q)+p}\right) p e^{-p u}:=C_{q} p e^{-p u}
$$

which means that the associated subordinator $\mathcal{N}_{2, q}$ is a compound Poisson process with intensity $C_{q}$ and jump sizes with density $f_{2}$. In this case we obtain an explicit expression for $F_{I_{e_{q}}}$ and its Laplace transform:

$$
\widehat{F}_{I_{e_{q}}}(r)=\frac{\frac{C_{q} p}{c}}{\frac{p\left(c-C_{q}\right)}{c}+r} \quad \text { and } \quad F_{I_{e_{q}}}(-x)=\frac{C_{q} p}{c} e^{-p\left(c-C_{q}\right) x}
$$

2. Now let us suppose that $\bar{F}_{2}(x)=\left(\frac{\theta}{\theta+x^{c}}\right)^{\xi}$ for $\xi, c, \theta>0$. This corresponds to a classical two-sided jumps risk process with claims given by a Burr distribution with parameters $\xi, \theta$ and $c$. Then

$$
\chi_{q, \mathcal{S}}(u)=\lambda_{2} \sum_{j=1}^{m+1} E(j, 0, q) \int_{u}^{\infty} e^{-\beta_{j}(q)(y-u)} \frac{\xi \theta^{\xi}}{(\theta+y)^{1+\xi}} d y
$$

and applying L'Hôpital's rule twice we get

$$
\lim _{u \rightarrow \infty} \frac{\bar{\chi}_{q, \mathcal{S}}(u)}{\left(\frac{\theta}{\theta+u^{c}}\right)^{\xi}}=\lambda_{2} \sum_{j=1}^{m+1} E(j, 0, q) \lim _{u \rightarrow \infty} \frac{\int_{u}^{\infty} e^{\beta_{j}(q) x} \int_{x}^{\infty} e^{-\beta_{j}(q) y} \frac{\xi \theta^{\xi}}{\left(\theta+y^{c}\right)^{1+\xi}} d y}{\left(\frac{\theta}{\theta+u^{c}}\right)^{\xi}}=\lambda_{2} \sum_{j=1}^{m+1} E(j, 0, q) \frac{1}{\beta_{j}(q)} .
$$

Using the second equality in Lemma 5.2 in Kolkovska and Martín-González [2016], it follows that

$$
\lim _{u \rightarrow \infty} \frac{\bar{\chi}_{q, \mathcal{S}}(u)}{\left(\frac{\theta}{\theta+u^{c}}\right)^{\xi}}=\lambda_{2} \prod_{i=1}^{N} \alpha_{i}^{n_{i}}\left(\prod_{j=1}^{m+1} \beta_{j}(q)\right)^{-1}=\frac{\lambda_{2} a(q)}{q}
$$

which implies that $1-\frac{\bar{\chi}_{q, \mathcal{S}}(u)}{\bar{\chi}_{q, \mathcal{S}}(0)}$ is a subexponential distribution. Therefore, due to Proposition 2 we obtain

$$
F_{I_{q}}(-u) \approx \frac{\lambda_{2}}{q}\left(\frac{\theta}{\theta+u^{c}}\right)^{\xi} \quad \text { as } \quad u \rightarrow \infty
$$


Example 3 (case C). Let us suppose that $\mathcal{S}$ is a spectrally positive $\xi$-stable process, with $\mathbb{E}[\mathcal{S}(1)]=0$ and $\xi \in(1,2)$. In this case $-\Psi_{\mathcal{S}}(r)=r^{\xi}$ and

$$
\chi_{q, \mathcal{S}}(x)=\frac{1}{\xi}\left(x^{-\xi}-\sum_{j=1}^{m+1} \beta_{j}(q) E(j, 0, q) \int_{x}^{\infty} e^{-\beta_{j}(q)(y-x)} y^{-\xi} d y\right),
$$

where we have used that $E_{*}(j, 0, q)=\beta_{j}(q) E(j, 0, q)$.

Notice that the function $\mathcal{L}_{\xi}(x)=\sum_{j=1}^{m+1} \beta_{j}(q) E(j, 0, q) \int_{x}^{\infty} e^{-\beta_{j}(q)(y-x)} y^{-\xi} d y$ coincides with the function $F_{\xi}$ defined in Kolkovska and Martín-González [2018], hence from Proposition 1 in the aforementioned work we obtain

$$
\int_{x}^{\infty} \mathcal{L}_{\xi}(y) d y \approx\left(1-\prod_{i=1}^{N} \alpha_{i}^{n_{i}}\left(\prod_{j=1}^{m+1} \beta_{j}(q)\right)^{-1}\right) \frac{x^{1-\xi}}{\Gamma(2-\xi)} \quad \text { as } \quad x \rightarrow \infty .
$$

Since $\prod_{i=1}^{N} \alpha_{i}^{n_{i}}\left(\prod_{j=1}^{m+1} \beta_{j}(q)\right)^{-1}=\frac{a(q)}{q}$, it follows that $\bar{\chi}_{q, \mathcal{S}}(x) \approx C_{\xi} \frac{x^{1-\xi}}{\Gamma(2-\xi)}$ as $x \rightarrow \infty$, where $C_{\xi}=$ $\frac{1}{\xi}\left(\frac{\Gamma(2-\xi)}{\xi-1}-1+\frac{a(q)}{q}\right)$. Therefore, from Proposition 2 we obtain $F_{I_{e_{q}}}(-u) \approx \frac{C_{\xi}}{a(q) \Gamma(2-\xi)} u^{1-\xi}$ as $u \rightarrow \infty$.

\section{Proof of Lemma 5}

This section is devoted to the proof of Lemma 5, It requires some preliminary results which we state first. Let $x_{1}, x_{2}, \ldots, x_{k}$ be different complex numbers. For $m \in\{1,2, \ldots\}$ let us denote $\left(x_{i}\right)_{m}=\underbrace{x_{i}, x_{i}, \ldots, x_{i}}_{m \text { times }}$, $i=1, \ldots, k$. The following result follows from standard tools in interpolation theory.

Lemma 8 Let $f: \mathbb{C} \rightarrow \mathbb{C}$ be an analytic function and define $g: \mathbb{C}^{m} \rightarrow \mathbb{C}$ by

$$
g\left[x_{1}, \ldots, x_{m}\right]=(-1)^{m-1} \sum_{j=1}^{m} \frac{f\left(x_{j}\right)}{\prod_{l \neq j}\left(x_{l}-x_{j}\right)},
$$

where $x_{i} \neq x_{j}$ for $i \neq j$. Let $n_{1}, n_{2}, \ldots, n_{k}$ be given natural numbers, and $m=\sum_{j=1}^{k} n_{j}$.

Then the function $g$ can be extended analytically for multiple points by the expression

$$
g\left[\left(x_{1}\right)_{n_{1}}, \ldots,\left(x_{k}\right)_{n_{k}}\right]=(-1)^{m-1} \sum_{j=1}^{k} \frac{(-1)^{m-n_{j}}}{\left(n_{j}-1\right) !} \frac{\partial^{n_{j}-1}}{\partial s^{n_{j}-1}}\left[\frac{f(s)\left(x_{j}-s\right)^{n_{j}}}{\prod_{l=1}^{k}\left(x_{l}-s\right)^{n_{l}}}\right]_{s=x_{j}} .
$$

We also need the following two lemmas. The first one is a well-known formula in interpolation theory, while the second one is part of the proof of Proposition 5.4 in Kolkovska and Martín-González [2016]. 
Lemma 9 Let $m \geq 1$ and let $a_{1}, \ldots, a_{m}$ be given different numbers. Then

$$
\sum_{j=1}^{m} \frac{\left(a_{j}-s\right)^{k}}{\prod_{l=1, l \neq j}^{m}\left(a_{l}-a_{j}\right)}= \begin{cases}(-1)^{m-1}, & k=m-1, \\ 0, & k=0,1, \ldots, m-2 \\ \frac{1}{\prod_{j=1}^{m}\left(a_{j}-s\right)}, & k=-1 .\end{cases}
$$

In what follows we set $P_{1}(r)=\prod_{j=1}^{N}\left(\alpha_{j}-r\right)^{n_{j}}$.

Lemma 10 Let $r, r_{1}, r_{2}, \ldots, r_{m+1}$ be $m+2$ different complex numbers and define

$$
J_{0}(r)=\lambda_{1} \sum_{j=1}^{m+1} P_{1}\left(r_{j}\right) \frac{1}{\prod_{k=1, i \neq j}^{m+1}\left(r_{k}-r_{j}\right)} \frac{\frac{Q(-r)}{\prod_{l=1}^{N}\left(\alpha_{l}-r\right)^{n_{l}}}-\frac{Q\left(-r_{j}\right)}{\prod_{l=1}^{N}\left(\alpha_{l}-r_{j}\right)^{n_{l}}}}{r_{j}-r} .
$$

Then $J_{0}(r)=0$ for all $r \in \mathbb{C}$.

The following result is used in case $\mathrm{C}$.

Lemma 11 Let $\nu_{\mathcal{S}}$ be the Lévy measure of a spectrally positive pure jump Lévy process such that

$$
\int_{0+}^{\infty}\left(x^{2} \wedge x\right) \nu_{\mathcal{S}}(d x)<\infty
$$

Then

$$
-\Psi_{\mathcal{S}}(r)=r \int_{0+}^{\infty}\left(1-e^{-r x}\right) \overline{\mathcal{V}}_{\mathcal{S}}(x) d x
$$

Moreover, for any $r_{1}, r_{2} \in \mathbb{C}_{+}$such that $r_{1} \neq r_{2}$ and $\Psi_{\mathcal{S}}$ exists, there holds

$$
\frac{\Psi_{\mathcal{S}}\left(r_{1}\right)-\Psi_{\mathcal{S}}\left(r_{2}\right)}{r_{2}-r_{1}}=r_{2} \widehat{T}_{r_{2}} \overline{\mathcal{V}}_{\mathcal{S}}\left(r_{1}\right)-\frac{\Psi_{\mathcal{S}}\left(r_{1}\right)}{r_{1}}=r_{1} \widehat{T}_{r_{2}} \overline{\mathcal{V}}_{\mathcal{S}}\left(r_{1}\right)-\frac{\Psi_{\mathcal{S}}\left(r_{2}\right)}{r_{2}}
$$

Proof. From assumption (6.2) we can restrict to $h \equiv 1$, and by applying Fubini's theorem we get (6.3). On the other hand, we have:

$$
\begin{aligned}
\frac{\Psi_{\mathcal{S}}\left(r_{1}\right)-\Psi_{\mathcal{S}}\left(r_{2}\right)}{r_{2}-r_{1}} & =\frac{r_{1} \frac{\Psi_{\mathcal{S}}\left(r_{1}\right)}{r_{1}}-r_{2} \frac{\Psi_{\mathcal{S}}\left(r_{2}\right)}{r_{2}}}{r_{2}-r_{1}}=\frac{r_{1}-r_{2}}{r_{2}-r_{1}} \frac{\Psi_{\mathcal{S}}\left(r_{1}\right)}{r_{1}}+r_{2} \frac{\frac{\Psi_{\mathcal{S}}\left(r_{1}\right)}{r_{1}}-\frac{\Psi_{\mathcal{S}}\left(r_{2}\right)}{r_{2}}}{r_{2}-r_{1}} \\
& =r_{2} \frac{\frac{\Psi_{\mathcal{S}}\left(r_{1}\right)}{r_{1}}-\frac{\Psi_{\mathcal{S}}\left(r_{2}\right)}{r_{2}}}{r_{2}-r_{1}}-\frac{\Psi_{\mathcal{S}}\left(r_{1}\right)}{r_{1}} .
\end{aligned}
$$

Similarly,

$$
\frac{\Psi_{\mathcal{S}}\left(r_{1}\right)-\Psi_{\mathcal{S}}\left(r_{2}\right)}{r_{2}-r_{1}}=r_{1} \frac{\frac{\Psi_{\mathcal{S}}\left(r_{1}\right)}{r_{1}}-\frac{\Psi_{\mathcal{S}}\left(r_{2}\right)}{r_{2}}}{r_{2}-r_{1}}-\frac{\Psi_{\mathcal{S}}\left(r_{2}\right)}{r_{2}}
$$


and

$$
\begin{aligned}
\frac{\Psi_{\mathcal{S}}\left(r_{1}\right)}{r_{1}}-\frac{\Psi_{\mathcal{S}}\left(r_{2}\right)}{r_{2}} & =\int_{0+}^{\infty}\left[\frac{1-e^{-r_{1} x}-r_{1} x}{r_{1}}-\frac{1-e^{-r_{2} x}-r_{2} x}{r_{2}}\right] \nu_{\mathcal{S}}(d x) \\
& =\int_{0+}^{\infty} \int_{0}^{x}\left[e^{-r_{1} y}-1-\left(e^{-r_{2} y}-1\right)\right] d y \nu_{\mathcal{S}}(d x)=\int_{0+}^{\infty} \int_{y}^{\infty} \nu_{\mathcal{S}}(d x)\left[e^{-r_{1} y}-e^{-r_{2} y}\right] d y \\
& =\widehat{\overline{\mathcal{V}}}_{\mathcal{S}}\left(r_{1}\right)-\widehat{\overline{\mathcal{V}}}_{\mathcal{S}}\left(r_{2}\right),
\end{aligned}
$$

where the third equality follows from Fubini's theorem. Substituting the last equality in (6.5) and (6.6), and using (2.4), we obtain the result.

Now we are ready to prove Lemma 5 .

Proof of Lemma 5, We deal only with the case $q>0$. The case $q=0$ follows by taking limits when $q \downarrow 0$ and that the $\operatorname{limit} \lim _{q \downarrow 0} \beta_{j}(q)$ exists and $\lim _{q \downarrow 0} \frac{q}{\beta_{1}(q)}=\mathbb{E}[\mathcal{X}(1)]$.

The proof of the lemma is simpler when the roots $\beta_{j}(q)$ of the generalized Lundberg equation are simple (see equality (6.11) below). In case of multiple roots we will approximate the Lundberg equation by an Lundberg equation depending on parameter $\epsilon$ which has simple roots, and such that when $\epsilon \rightarrow 0$, the roots of the approximating Lundberg equation approximate the multiple roots of the given equation. At the end of the proof we take $\epsilon \rightarrow 0$ to obtain Lemma 5 in case of multiple roots $\beta_{j}(q)$.

First we obtain the result for the case $\mathrm{C}$. Recall that $\beta_{j}(q)$ are the roots of the generalized Lundberg function of $\mathcal{X}$. Let $\varepsilon \in(0, E)$, where

$$
E=\min \left\{\left|\operatorname{Re}\left(\beta_{i}(q)\right)-\operatorname{Re}\left(\beta_{j}(q)\right)\right|: \operatorname{Re}\left(\beta_{i}(q)\right)-\operatorname{Re}\left(\beta_{j}(q)\right) \neq 0\right\},
$$

and define the complex numbers

$$
\begin{aligned}
& \beta_{1}(q)^{*}=\beta_{1}(q), \beta_{2}(q)^{*}=\beta_{2}(q)+\frac{1}{m+1} \varepsilon, \ldots, \beta_{k_{1}}(q)^{*}=\beta_{1}(q)+\frac{k_{1}-1}{m+1} \varepsilon, \\
& \beta_{k_{1}+1}(q)^{*}=\beta_{2}(q), \ldots, \beta_{k_{1}+k_{2}}(q)^{*}=\beta_{2}(q)+\frac{k_{1}+k_{2}-1}{m+1} \varepsilon \\
& \beta_{k_{1}+\cdots+k_{R-1}+1}(q)^{*}=\beta_{R}(q), \ldots, \beta_{m+1}(q)^{*}=\beta_{R}(q)+\frac{j}{m+1} \varepsilon
\end{aligned}
$$

where we have omitted the dependence on $\varepsilon$ for simplicity. It follows that $\lim _{\varepsilon \rightarrow 0} \beta_{l_{j}+a_{j}}(q)^{*}=\beta_{j}(q), j=$ $1,2, \ldots, R$, for $l_{1}=0, l_{2}=k_{1}, \ldots, l_{R}=k_{R-1}$ and $a_{j}=1,2, \ldots, k_{j}$.

This gives $m+1$ different numbers $\beta_{1}^{*}, \ldots, \beta_{m+1}^{*}$ such that, as $\varepsilon \downarrow 0$, the first $k_{1}$ numbers converge to $\beta_{1}(q)$, the next $k_{2}$ numbers converge to $\beta_{2}(q)$, and so on.

From the definition of $\Psi_{\mathcal{X}}$,

$$
\Psi_{\mathcal{X}}\left[\beta_{j}(q)^{*}\right]-q=-\Psi_{\mathcal{S}}\left[\beta_{j}(q)^{*}\right]+\lambda_{1} \frac{Q\left(-\beta_{j}(q)^{*}\right)}{\prod_{l=1}^{N}\left(\alpha_{l}-\beta_{j}(q)^{*}\right)^{n_{l}}}+c \beta_{j}(q)^{*}+\gamma^{2}\left[\beta_{j}(q)^{*}\right]^{2}-q-\lambda_{1} .
$$

Therefore, for each $j=1,2, \ldots, m+1$ we obtain

$$
\lambda_{1}+q=-\Psi_{\mathcal{S}}\left[\beta_{j}(q)^{*}\right]+\lambda_{1} \frac{Q\left(-\beta_{j}(q)^{*}\right)}{\prod_{l=1}^{N}\left(\alpha_{l}-\beta_{j}(q)^{*}\right)^{n_{l}}}+c \beta_{j}(q)^{*}+\gamma^{2}\left[\beta_{j}(q)^{*}\right]^{2}-\left(\Psi_{\mathcal{X}}\left[\beta_{j}(q)^{*}\right]-q\right),
$$


which yields

$$
\begin{aligned}
\Psi_{\mathcal{X}}(r)-q & =-\Psi_{\mathcal{S}}(r)+\lambda_{1} \frac{Q(-r)}{\prod_{l=1}^{N}\left(\alpha_{l}-r\right)^{n_{l}}}+c r+\gamma^{2} r^{2}+\Psi_{\mathcal{S}}\left[\beta_{j}(q)^{*}\right]-\lambda_{1} \frac{Q\left(-\beta_{j}(q)^{*}\right)}{\prod_{l=1}^{N}\left(\alpha_{l}-\beta_{j}(q)^{*}\right)^{n_{l}}} \\
& -c \beta_{j}(q)^{*}-\gamma^{2}\left[\beta_{j}(q)^{*}\right]^{2}+\Psi_{\mathcal{X}}\left[\beta_{j}(q)^{*}\right]-q .
\end{aligned}
$$

Since $P_{1}$ is a polynomial with degree $m$, using Lagrange interpolation we obtain the equivalent representation

$$
P_{1}(r)=\sum_{l=1}^{m+1} \frac{\prod_{j=1}^{N}\left[\alpha_{j}-\beta_{l}(q)^{*}\right]^{n_{j}}}{\prod_{j \neq l}\left[\beta_{j}(q)^{*}-\beta_{l}(q)^{*}\right]} \prod_{j \neq l}\left[\beta_{j}(q)^{*}-r\right] .
$$

This and (6.8) give

$$
\begin{aligned}
{\left[\Psi_{\mathcal{X}}(r)-q\right] P_{1}(r) } & =\sum_{l=1}^{m+1} \frac{P_{1}\left[\beta_{l}(q)^{*}\right]}{\prod_{j \neq l}\left[\beta_{j}(q)^{*}-\beta_{l}(q)^{*}\right]} \prod_{j \neq l}\left[\beta_{j}(q)^{*}-r\right]\left\{-\left(\beta_{l}(q)^{*}-r\right) \frac{\Psi_{\mathcal{S}}(r)-\Psi_{\mathcal{S}}\left(\beta_{l}(q)^{*}\right)}{\beta_{l}(q)^{*}-r}\right. \\
& \left.+\left(\beta_{l}(q)^{*}-r\right)\left(\lambda_{1} J_{0}^{*}-c-\gamma^{2}\left(\beta_{l}(q)^{*}+r\right)\right)+\Psi_{\mathcal{X}}\left(\beta_{l}(q)^{*}\right)-q\right\} \\
& =\prod_{j=1}^{m+1}\left[\beta_{j}(q)^{*}-r\right] \sum_{l=1}^{m+1} \frac{P_{1}\left[\beta_{l}(q)^{*}\right]}{\prod_{j \neq l}\left[\beta_{j}(q)^{*}-\beta_{l}(q)^{*}\right]}\left\{-\frac{\Psi_{\mathcal{S}}(r)-\Psi_{\mathcal{S}}\left(\beta_{l}(q)^{*}\right)}{\beta_{l}(q)^{*}-r}\right. \\
& \left.+\lambda_{1} J_{0}^{*}-c-\gamma^{2}\left(\beta_{l}(q)^{*}+r\right)+\frac{\Psi_{\mathcal{X}}\left(\beta_{l}(q)^{*}\right)-q}{\beta_{l}(q)^{*}-r}\right\}
\end{aligned}
$$

where $J_{0}^{*}=\frac{\frac{Q(-r)}{\prod_{j=1}^{N}\left(\alpha_{j}-r\right)^{n_{j}}}-\frac{Q\left(-\beta_{l}(q)^{*}\right)}{\prod_{j=1}^{N}\left(\alpha_{j}-\beta_{l}(q)^{*}\right)^{n_{j}}}}{\beta_{l}(q)^{*}-r}$. Formula 6.1) and Lemma10 imply, respectively:

$$
\sum_{l=1}^{m+1} \frac{P_{1}\left[\beta_{l}(q)^{*}\right]}{\prod_{j \neq l}\left[\beta_{j}(q)^{*}-\beta_{l}(q)^{*}\right]}=1 \quad \text { and } \quad \lambda_{1} \sum_{l=1}^{m+1} \frac{P_{1}\left[\beta_{l}(q)^{*}\right]}{\prod_{j \neq l}\left[\beta_{j}(q)^{*}-\beta_{l}(q)^{*}\right]} J_{0}^{*}=0
$$

Substituting these two equalities in $(6.9)$, using the first equality in $(6.4)$ to calculate $\frac{\Psi_{\mathcal{S}}(r)-\Psi_{\mathcal{S}}\left(\beta_{j}(q)^{*}\right)}{\beta_{j}(q)^{*}-r}$ and dividing by $\prod_{j=1}^{m+1}\left[\beta_{j}(q)^{*}-r\right]$, we obtain

$$
\begin{aligned}
& {\left[\Psi_{\mathcal{X}}(r)-q\right] \frac{P_{1}(r)}{\prod_{j=1}^{m+1}\left[\beta_{j}(q)^{*}-r\right]}} \\
& =\sum_{l=1}^{m+1} \frac{P_{1}\left[\beta_{l}(q)^{*}\right]}{\prod_{j \neq l}\left[\beta_{j}(q)^{*}-\beta_{l}(q)^{*}\right]}\left\{-\beta_{l}(q)^{*} \widehat{T}_{\beta_{l}(q)^{*}} \overline{\mathcal{V}}_{\mathcal{S}}(r)+\frac{\Psi_{\mathcal{S}}(r)}{r}-c-\gamma^{2}\left[r+\beta_{l}(q)^{*}\right]+\frac{\Psi_{\mathcal{X}}\left[\beta_{l}(q)^{*}\right]-q}{\beta_{j}(q)^{*}-r}\right\} \\
& =\frac{\Psi_{\mathcal{S}}(r)}{r}-c-\gamma^{2} r-\sum_{l=1}^{m+1} \frac{P_{1}\left[\beta_{l}(q)^{*}\right]}{\prod_{j \neq l}\left[\beta_{j}(q)^{*}-\beta_{l}(q)^{*}\right]}\left\{\beta_{l}(q)^{*} \widehat{T}_{\beta_{l}(q)^{*}} \overline{\mathcal{V}}_{\mathcal{S}}(r)+\gamma^{2} \beta_{l}(q)^{*}-\frac{\Psi_{\mathcal{X}}\left[\beta_{l}(q)^{*}\right]-q}{\beta_{j}(q)^{*}-r}\right\},
\end{aligned}
$$


where the last equality follows from 6.10 .

Using Lemmaß3 and Theorem 2.2 in Lewis and Mordecki [2008] we obtain $\lim _{\varepsilon \downarrow 0} \frac{P_{1}(r)}{\prod_{j=1}^{m+1}\left[\beta_{j}(q)^{*}-r\right]}=(\kappa(q,-r))^{-1}$.

Hence we let $\varepsilon \downarrow 0$ in both sides of 6.11 and apply Lemma 8 , This yields:

$$
\begin{aligned}
& {\left[\Psi_{\mathcal{X}}(r)-q\right](\kappa(q,-r))^{-1}} \\
& =\frac{\Psi_{\mathcal{S}}(r)}{r}-c-\gamma^{2} r-\sum_{l=1}^{R} \frac{(-1)^{1-k_{l}}}{\left(k_{l}-1\right) !} \frac{\partial^{k_{l}-1}}{\partial s^{k_{l}-1}}\left[\frac{\prod_{j=1}^{N}\left(\alpha_{j}-s\right)^{n_{j}}\left(\beta_{l}(q)-s\right)^{k_{l}}}{\prod_{j=1}^{R}\left(\beta_{j}(q)-s\right)^{k_{j}}} \widehat{T}_{s} \overline{\mathcal{V}}_{\mathcal{S}}(r)\right]_{s=\beta_{l}(q)} \\
& -\gamma^{2} \sum_{l=1}^{R} \frac{(-1)^{1-k_{l}}}{\left(k_{l}-1\right) !} \frac{\partial^{k_{l}-1}}{\partial s^{k_{j}-1}}\left[\frac{\prod_{j=1}^{N}\left(\alpha_{j}-s\right)^{n_{j}}\left(\beta_{l}(q)-s\right)^{k_{l}}}{\prod_{j=1}^{R}\left(\beta_{j}(q)-s\right)^{k_{j}}}\right]_{s=\beta_{l}(q)} \\
& +\sum_{l=1}^{R} \frac{(-1)^{1-k_{l}}}{\left(k_{l}-1\right) !} \frac{\partial^{k_{l}-1}}{\partial s^{k_{l}-1}}\left[\frac{\prod_{j=1}^{N}\left(\alpha_{j}-s\right)^{n_{j}}\left(\beta_{l}(q)-s\right)^{k_{l}}}{\prod_{j=1}^{R}\left(\beta_{j}(q)-s\right)^{k_{j}}} \frac{\Psi_{\mathcal{X}}(s)-q}{s-r}\right]_{s=\beta_{l}(q)} .
\end{aligned}
$$

Since, for $j=1,2, \ldots, R, \beta_{j}(q)$ are roots of $\Psi_{\mathcal{X}}(s)-q=0$ in $\mathbb{C}_{++}$with respective multiplicities $k_{j}$, it follows from the Leibniz rule that

$$
\sum_{l=1}^{R} \frac{(-1)^{1-k_{l}}}{\left(k_{l}-1\right) !} \frac{\partial^{k_{l}-1}}{\partial s^{k_{l}-1}}\left[\frac{P_{1}(s)\left(\beta_{l}(q)-s\right)^{k_{l}}}{\prod_{j=1}^{R}\left(\beta_{j}(q)-s\right)^{k_{j}}} \frac{\Psi_{\mathcal{X}}(s)-q}{s-r}\right]_{s=\beta_{l}(q)}=0
$$

Hence, substituting this in the equality above and setting $D_{q}=\sum_{l=1}^{R} \frac{(-1)^{1-k_{l}}}{\left(k_{l}-1\right) !} \frac{\partial^{k_{l}-1}}{\partial s^{k_{j}-1}}\left[\frac{\prod_{j=1}^{N}\left(\alpha_{j}-s\right)^{n_{j}}\left(\beta_{l}(q)-s\right)^{k_{l}}}{\prod_{j=1}^{R}\left(\beta_{j}(q)-s\right)^{k_{j}}} s\right]_{s=\beta_{l}(q)}$, we obtain:

$$
\begin{aligned}
& {\left[q-\Psi_{\mathcal{X}}(r)\right](\kappa(q,-r))^{-1}} \\
& =c+\gamma^{2} D_{q}+\gamma^{2} r-\frac{\Psi_{\mathcal{S}}(r)}{r}+\sum_{l=1}^{R} \frac{(-1)^{1-k_{l}}}{\left(k_{l}-1\right) !} \frac{\partial^{k_{l}-1}}{\partial s^{k_{l}-1}}\left[\frac{\prod_{j=1}^{N}\left(\alpha_{j}-s\right)^{n_{j}}\left(\beta_{l}(q)-s\right)^{k_{l}}}{\prod_{j=1}^{R}\left(\beta_{j}(q)-s\right)^{k_{j}}} \widehat{T}_{s} \overline{\mathcal{V}}_{\mathcal{S}}(r)\right]_{s=\beta_{l}(q)} .
\end{aligned}
$$

Using Leibniz rule and Lemma2 we get

$$
\sum_{l=1}^{R} \sum_{a=0}^{k_{l}-1}\left(\begin{array}{c}
k_{l}-1 \\
a
\end{array}\right) \frac{(-1)^{1-k_{l}}}{\left(k_{l}-1\right) !} \frac{\partial^{k_{l}-1-a}}{\partial s^{k_{l}-1-a}}\left[\frac{\prod_{j=1}^{N}\left(\alpha_{j}-s\right)^{n_{j}}\left(\beta_{l}(q)-s\right)^{k_{l}}}{\prod_{j=1}^{R}\left(\beta_{j}(q)-s\right)^{k_{j}}} s\right]_{s=\beta_{l}(q)} \frac{\partial^{a}}{\partial s^{a}}\left[\widehat{T}_{s} \overline{\mathcal{V}}_{\mathcal{S}}(r)\right]_{s=\beta_{l}(q)}
$$




$$
=\sum_{l=1}^{R} \sum_{a=0}^{k_{l}-1} E_{*}(l, a, q) \widehat{\mathcal{T}}_{\beta_{l}(q), a} \overline{\mathcal{V}}_{\mathcal{S}}(r)=\sum_{l=1}^{R} \sum_{a=0}^{k_{l}-1} E_{*}(l, a, q) \widehat{\mathcal{T}}_{\beta_{l}(q), a} \overline{\mathcal{V}}_{\mathcal{S}}(r)=\widehat{\mathcal{L}}_{q}(r)
$$

hence from (6.12) we obtain

$$
\left[q-\Psi_{\mathcal{X}}(r)\right](\kappa(q,-r))^{-1}=c+\gamma^{2} D_{q}+\gamma^{2} r-\frac{\Psi_{\mathcal{S}}(r)}{r}+\widehat{\mathcal{L}}_{q}(r) .
$$

Since by L'Hôpital's rule $\lim _{r \downarrow 0} \frac{\Psi_{\mathcal{S}}(r)}{r}=0$, it follows that $a(q)=c+\gamma^{2} D_{q}+\widehat{\mathcal{L}}_{q}(0)$. Hence

$$
\begin{aligned}
{\left[q-\Psi_{\mathcal{X}}(r)\right](\kappa(q,-r))^{-1} } & =a(q)-\widehat{\mathcal{L}}_{q}(0)+\gamma^{2} r-\frac{\Psi_{\mathcal{S}}(r)}{r}+\widehat{\mathcal{L}}_{q}(r) \\
& =a(q)+\gamma^{2} r-\frac{\Psi_{\mathcal{S}}(r)}{r}-\left(\widehat{\mathcal{L}}_{q}(0)-\widehat{\mathcal{L}}_{q}(r)\right),
\end{aligned}
$$

and we obtain the result for case $\mathrm{C}$.

To obtain the result for case $\mathrm{B}$, we use the same notations as above for the $m+1$ roots of the generalized Lundberg equation, and use (6.9) with $\Psi_{\mathcal{S}}(r)$ replaced by $G_{\mathcal{S}}(r)$ and setting $\gamma=0$. This gives:

$$
\begin{aligned}
& {\left[\Psi_{\mathcal{X}}(r)-q\right] P_{1}(r)} \\
& =\prod_{j=1}^{m+1}\left[\beta_{j}(q)^{*}-r\right] \sum_{l=1}^{m+1} \frac{P_{1}\left[\beta_{l}(q)^{*}\right]}{\prod_{j \neq l}\left[\beta_{j}(q)^{*}-\beta_{l}(q)^{*}\right]}\left\{-\frac{G_{\mathcal{S}}(r)-G_{\mathcal{S}}\left(\beta_{l}(q)^{*}\right)}{\beta_{l}(q)^{*}-r}+\lambda_{1} J_{0}^{*}-c+\frac{\Psi_{\mathcal{X}}\left(\beta_{l}(q)^{*}\right)-q}{\beta_{l}(q)^{*}-r}\right\} \\
& =-c+\prod_{j=1}^{m+1}\left[\beta_{j}(q)^{*}-r\right] \sum_{l=1}^{m+1} \frac{P_{1}\left[\beta_{l}(q)^{*}\right]}{\prod_{j \neq l}\left[\beta_{j}(q)^{*}-\beta_{l}(q)^{*}\right]}\left\{\widehat{T}_{\left.\beta_{l}(q)^{*} \nu_{\mathcal{S}}(r)+\frac{\Psi_{\mathcal{X}}\left(\beta_{l}(q)^{*}\right)-q}{\beta_{l}(q)^{*}-r}\right\},}\right.
\end{aligned}
$$

where in the second equality we used (6.10) and the fact that $\frac{G_{\mathcal{S}}(r)-G_{\mathcal{S}}(s)}{s-r}=-\widehat{T}_{s} \nu_{\mathcal{S}}(r)$, which follows from the second equality in (2.4). Proceeding as in case $\mathrm{C}$, we obtain

$$
\begin{aligned}
& {\left[q-\Psi_{\mathcal{X}}(r)\right](\kappa(q,-r))^{-1}} \\
& =c-\sum_{l=1}^{R} \sum_{a=0}^{k_{l}-1}\left(\begin{array}{c}
k_{l}-1 \\
a
\end{array}\right) \frac{(-1)^{1-k_{l}+a}}{\left(k_{l}-1\right) !} \frac{\partial^{k_{l}-1-a}}{\partial s^{k_{l}-1-a}}\left[\frac{\prod_{j=1}^{N}\left(\alpha_{j}-s\right)^{n_{j}}\left(\beta_{l}(q)-s\right)^{k_{l}}}{\prod_{j=1}^{R}\left(\beta_{j}(q)-s\right)^{k_{j}}}\right]_{s=\beta_{l}(q)} \widehat{\mathcal{T}}_{\beta_{l}(q), a} \nu_{\mathcal{S}}(r) \\
& =c-\widehat{\ell}_{q}(r),
\end{aligned}
$$

Now we set $r=0$ in the above equality to obtain

$$
c=a(q)+\widehat{\ell}_{q}(0)
$$

This gives the result for case $\mathrm{B}$.

In case A we have $\Psi_{\mathcal{X}}(r)=\lambda_{1}\left(\widehat{f}_{1}(-r)-1\right)-G_{\mathcal{S}}(r)$. For now we assume that $\mathcal{S}$ is not a compound Poisson process. In this case we know from Lemma 1 that the equation $\Psi_{\mathcal{X}}(r)-q=0$ has only $m$ 
roots in $\mathbb{C}_{++}$, denoted as before and such that they have respective multiplicities $k_{1} \equiv 1, k_{2}, \ldots, k_{R}$, where $\sum_{j=1}^{R} k_{j}=m$. Now we consider the numbers $\beta_{1}^{*}(q), \ldots, \beta_{m}(q)^{*}$ as defined in 6.7) with $\beta_{m+1}(q)^{*}$ replaced by $\beta_{\infty}(n)=\sqrt{n}$. We also take $c_{n}=\frac{1}{n}$. In this case, the function $\Psi_{\mathcal{X}}(r)+c_{n} r$ is the generalized Lundberg function of some Lévy process of the form (2.1) with $\gamma=0$ and drift term $c_{n}$. Hence we can use (6.9) with $\gamma=0, G_{\mathcal{S}}$ instead of $\Psi_{\mathcal{S}}$ and $c_{n}$ instead of $c$. This gives

$$
\begin{aligned}
& {\left[\Psi_{\mathcal{X}}(r)+c_{n} r-q\right] P_{1}(r)} \\
& =\left(\beta_{\infty}(n)-r\right) \prod_{k=1}^{m}\left[\rho_{k, q}^{*}-r\right] \sum_{l=1}^{m} \frac{P_{1}\left[\beta_{l}(q)^{*}\right]}{\left(\beta_{\infty}(n)-\beta_{l}(q)^{*}\right) \prod_{j \neq l}\left[\beta_{j}(q)^{*}-\beta_{l}(q)^{*}\right]}\left\{-\frac{G_{\mathcal{S}}(r)-G_{\mathcal{S}}\left(\beta_{l}(q)^{*}\right)}{\beta_{l}(q)^{*}-r}\right. \\
& \left.+\lambda_{1} \frac{\frac{Q(-r)}{\prod_{a=1}^{N}\left(\alpha_{a}-r\right)^{n_{a}}}-\frac{Q\left(-\beta_{l}(q)^{*}\right)}{\prod_{a=1}^{N}\left(\alpha_{a}-\beta_{l}(q)^{*}\right)^{n_{a}}}}{\beta_{l}(q)^{*}-r}-c_{n}+\frac{\Psi \mathcal{X}\left(\beta_{l}(q)^{*}\right)-q}{\beta_{l}(q)^{*}-r}\right\} \\
& +\left(\beta_{\infty}(n)-r\right) \prod_{k=1}^{m}\left[\rho_{k, q}^{*}-r\right] \frac{P_{1}\left[\beta_{\infty}(n)\right]}{\prod_{j=1}^{m}\left[\beta_{j}(q)^{*}-\beta_{\infty}(n)\right]}\left\{-\frac{G_{\mathcal{S}}(r)-G_{\mathcal{S}}\left(\beta_{\infty}(n)\right)}{\beta_{l}(q)^{*}-r}\right. \\
& \left.+\lambda_{1} \frac{\frac{Q(-r)}{\prod_{a=1}^{N}\left(\alpha_{a}-r\right)^{n_{a}}}-\frac{Q\left(-\beta_{\infty}(n)\right)}{\prod_{a=1}^{N}\left(\alpha_{a}-\beta_{\infty}(n)\right)^{n_{a}}}}{\beta_{\infty}(n)-r}-c_{n}+\frac{\Psi_{\mathcal{X}}\left(\beta_{\infty}(n)\right)-q}{\beta_{\infty}(n)-r}\right\}
\end{aligned}
$$

Again, proceeding as in case $\mathrm{C}$ it follows that

$$
\begin{aligned}
& {\left[\Psi_{\mathcal{X}}(r)+c_{n} r-q\right] \frac{P_{1}(r)}{\prod_{j=1}^{m}\left[\beta_{j}(q)^{*}-r\right]}=-c_{n}\left(\beta_{\infty}(n)-r\right)} \\
& +\sum_{l=1}^{m} \frac{\beta_{\infty}(n)-r}{\beta_{\infty}(n)-\beta_{l}(q)^{*}} \frac{P_{1}\left[\beta_{l}(q)^{*}\right]}{\prod_{j \neq l}\left[\beta_{j}(q)^{*}-\beta_{l}(q)^{*}\right]}\left\{\widehat{T}_{\left.\beta_{l}(q)^{*} \nu_{\mathcal{S}}(r)+\frac{\Psi_{\mathcal{X}}\left[\beta_{l}(q)^{*}\right]-q}{\beta_{l}(q)^{*}-r}\right\}}\right. \\
& +\frac{P_{1}\left[\beta_{\infty}(n)\right]}{\prod_{j=1}^{m}\left[\beta_{j}(q)^{*}-\beta_{\infty}(n)\right]}\left\{\left(\beta_{\infty}(n)-r\right) \widehat{T}_{\beta_{\infty}(n)} \nu_{\mathcal{S}}(r)+\left(\Psi_{\mathcal{X}}\left[\beta_{\infty}(n)\right]-q\right)\right\} \\
& =-c_{n}\left(\beta_{\infty}(n)-r\right)+\sum_{l=1}^{m} \frac{\beta_{\infty}(n)-r}{\beta_{\infty}(n)-\beta_{l}(q)^{*}} \frac{P_{1}\left[\beta_{l}(q)^{*}\right]}{\prod_{j \neq l}\left[\beta_{j}(q)^{*}-\beta_{l}(q)^{*}\right]}\left\{\widehat{T}_{\beta_{l}(q)^{*} \nu_{\mathcal{S}}(r)}\right. \\
& \left.+\frac{\Psi_{\mathcal{X}}\left[\beta_{l}(q)^{*}\right]-q}{\beta_{l}(q)^{*}-r}\right\}+\frac{P_{1}\left[\beta_{\infty}(n)\right]}{\prod_{j=1}^{m}\left[\beta_{j}(q)^{*}-\beta_{\infty}(n)\right]}\left\{-G_{\mathcal{S}}(r)+G_{\mathcal{S}}\left(\beta_{\infty}(n)\right)\right. \\
& \left.+\left(\Psi_{\mathcal{X}}\left[\beta_{\infty}(n)\right]-q\right)\right\},
\end{aligned}
$$

where in the second equality we have used that $\left(\beta_{\infty}(n)-r\right) \widehat{T}_{\beta_{\infty}(n)} \nu_{S}(r)=\left(\beta_{\infty}(n)-r\right)\left(\frac{-G_{\mathcal{S}}(r)+G_{\mathcal{S}}\left(\beta_{\infty}(n)\right)}{\beta_{\infty}(n)-r}\right)$. Now we substitute $\Psi_{\mathcal{X}}\left(\beta_{\infty}(n)\right)=\lambda_{1}\left(\frac{Q\left(-\beta_{\infty}(n)\right)}{\prod_{a=1}^{N}\left(\alpha_{a}-\beta_{\infty}(n)\right)^{n a}}-1\right)+c_{n} \beta_{\infty}(n)-G_{\mathcal{S}}\left(\beta_{\infty}(n)\right)$ in the above 
equality and rearrange terms to obtain

$$
\begin{aligned}
& {\left[\Psi_{\mathcal{X}}(r)+c_{n} r-q\right] \frac{P_{1}(r)}{\prod_{j=1}^{m}\left[\beta_{j}(q)^{*}-r\right]}} \\
& =-c_{n}\left(\beta_{\infty}(n)-r\right)+\sum_{l=1}^{m} \frac{\beta_{\infty}(n)-r}{\beta_{\infty}(n)-\beta_{l}(q)^{*}} \frac{P_{1}\left[\beta_{l}(q)^{*}\right]}{\prod_{j \neq l}\left[\beta_{j}(q)^{*}-\beta_{l}(q)^{*}\right]}\left\{\widehat{T}_{\beta_{l}(q) *} \nu_{\mathcal{S}}(r)\right. \\
& \left.+\frac{\Psi_{\mathcal{X}}\left[\beta_{l}(q)^{*}\right]-q}{\beta_{l}(q)^{*}-r}\right\}-\frac{P_{1}\left[\beta_{\infty}(n)\right]}{\prod_{j=1}^{m}\left[\beta_{j}(q)^{*}-\beta_{\infty}(n)\right]} G_{\mathcal{S}}(r) \\
& +\frac{P_{1}\left[\beta_{\infty}(n)\right]}{\prod_{j=1}^{m}\left[\beta_{j}(q)^{*}-\beta_{\infty}(n)\right]}\left\{c_{n} \beta_{\infty}(n)+\lambda_{1} \frac{Q\left(-\beta_{\infty}(n)\right)}{\prod_{a=1}^{N}\left(\alpha_{a}-\beta_{\infty}(n)\right)^{n_{a}}}-\left(\lambda_{1}+q\right)\right\} .
\end{aligned}
$$

Since both polynomials $P_{1}(r)=\prod_{j=1}^{N}\left(\alpha_{j}-r\right)^{n_{j}}$ and $\prod_{j=1}^{m}\left(\beta_{j}(q)^{*}-r\right)$ have degree $m$, and since $Q(r)$ given in 2.2 is a polynomial of degree at most $m-1$, it follows that for any fixed $r$ and $s$ such that $r \neq \beta_{\infty}(n)$ and $s \neq \beta_{\infty}(n)$,

$$
\lim _{n \rightarrow \infty} \frac{\beta_{\infty}(n)-r}{\beta_{\infty}(n)-s}=1, \quad \lim _{n \rightarrow \infty} \frac{P_{1}\left(\beta_{\infty}(n)\right)}{\prod_{j=1}^{m}\left(\beta_{j}(q)^{*}-\beta_{\infty}(n)\right)}=1, \quad \lim _{n \rightarrow \infty} \frac{Q\left(-\beta_{\infty}(n)\right)}{\prod_{a=1}^{N}\left(\alpha_{a}-\beta_{\infty}(n)\right)^{n_{a}}}=0 .
$$

Hence, letting $n \rightarrow \infty$ in both sides of (6.14) we get

$$
\begin{aligned}
& {\left[q-\Psi_{\mathcal{X}}(r)\right] \frac{\prod_{j=1}^{N}\left(\alpha_{j}-r\right)^{n_{j}}}{\prod_{j=1}^{m}\left(\beta_{j}(q)^{*}-r\right)}} \\
& =-\sum_{l=1}^{m} \frac{P_{1}\left[\beta_{l}(q)^{*}\right]}{\prod_{j \neq l}\left[\beta_{j}(q)^{*}-\beta_{l}(q)^{*}\right]}\left\{\widehat{T}_{\beta_{l}(q)^{*}} \nu_{\mathcal{S}}(r)+\frac{\Psi_{\mathcal{X}}\left[\beta_{l}(q)^{*}\right]-q}{\beta_{l}(q)^{*}-r}\right\}+G_{\mathcal{S}}(r)+\lambda_{1}+q .
\end{aligned}
$$

The remaining part of the proof is done similarly as in cases B and C using Lemma 3 , The case when $\mathcal{S}$ is a compound Poisson process with $\mathbb{E}[\mathcal{S}(1)]>0$ is obtained from case B as follows:

when $c=0$ we have $\left.\Psi_{\mathcal{X}}^{\prime}(r)\right|_{r=0+}=\lambda_{1} \mu_{1}-\lambda_{2} \mu_{2}$, hence $\psi_{\mathcal{X}}(r)-q=0$ has $m$ roots under the assumption that $\lambda_{1} \mu_{1}-\lambda_{2} \mu_{2}>0$.

If $\mathcal{X}_{c}$ denotes the process $\mathcal{X}$ with drift $c>0$ when $\mathcal{S}$ is a subordinator and $\mathcal{X}$ denotes the same process with $c=0$ we have $\psi_{\mathcal{X}_{c}}(r) \rightarrow \psi_{\mathcal{X}}(r)$, for all $r>0$ when $c \downarrow 0$.

This implies that the roots of $\psi_{\mathcal{X}_{c}}(r)-q$ must converge to those of $\psi_{\mathcal{X}_{c}}(r)-q$, but since the latter function has only $m$ roots, it must hold that one of the roots of $\psi_{\mathcal{X}_{c}}(r)-q$ (say $\beta_{m+1}(q)$ ) must converge to infinite when $c \downarrow 0$. Hence the result is obtained by the same procedure as before, replacing $c_{n}$ by $c$ and $\beta_{\infty}(n)$ by $\beta_{m+1}(q)$.

Acknowledment The authors are grateful to two anonymous referees for their careful reading of the paper and for many useful suggestions which greatly improved the presentation of the results. The first-named author appreciates partial support from CONACyT Grant No. 257867. 


\section{References}

S. Asmussen, F. Avram and M.R. Pistorius. Russian and American put options under exponential phase-type Lévy models. Stochastic Processes and their Applications, 109 (1), 79-111, 2004.

J. Bertoin. Lévy processes. Cambridge University Press, 1996.

A. Borovkov. Stochastic processes in queueing theory. Springer-Verlag New York, 1976.

D.C.M. Dickson and C. Hipp. On the time to ruin for Erlang(2) risk processes. Insurance: Mathematics and Economics, 29 (3), 333-344, 2001.

P. Embrechts, C. Goldie, and N. Veraverbeke. Subexponentiality and infinite divisibility. Zeitschrift für Wahrscheinlichkeitstheorie und Verwandte Gebiete, 49 (3), 335-347, 1979.

W. Feller. An introduction to probability theory and its applications, volume II. Wiley and Sons, 1971.

H.U. Gerber and E.S.W. Shiu. On the time value of ruin. North American Actuarial Journal, 2 (1), 48-78, 1998.

E.T. Kolkovska and E.M. Martín-González. Path functionals of a class of Lévy insurance risk processes Communications on Stochastic Analysis, 10(3), 363-387, 2016.

E.T. Kolkovska and E.M. Martín-González. Asymptotic behavior of the ruin probability, the severity of ruin and the surplus prior to ruin of a two-sided jumps perturbed risk process. XII Symposium of Probability and Stochastic Processes, Progress in Probability, p. 107-134, Birkhäuser, 2018.

A. Kuznetsov. Wiener-Hopf factorization and distribution of extrema for a family of Lévy processes. Annals of Applied Probability, 20 (5), 1801-1830, 2010a.

A. Kuznetsov. Wiener-Hopf factorization for a family of Lévy processes related to theta functions. Journal of Applied Probability, 47(4):1023-1033, 2010b.

A. Kuznetsov and X. Peng. On the Wiener-Hopf factorization for Lévy processes with bounded positive jumps. Stochastic Processes and Their Applications, 122(7):2610-2638, 2012.

A. Kyprianou. Introductory lectures on fluctuations of Lévy processes with applications. Springer-Verlag, Berlin Heidelberg, 2006.

A. Lewis and E. Mordecki. Wiener-hopf factorization for lévy processes having positive jumps with rational transforms. Journal of Applied Probability, 118-134, 45 (1), 2008.

T. Rolski, H. Schmidli, V. Schmidt, and J. Teugels. Stochastic Processes for Insurance and Finance. John Wiley and sons, 1999.

K. Sato. Lévy Processes and Infinitely Divisible Distributions. Cambridge University Press, 1999. 